kuLTura - meDia- TeoLogia

ISSN 2081-8971

$2017 \mathrm{nr} 28$, s. 91-107.

\title{
Lo sguardo di Salvatore Privitera sulla fecondazione assistita nel confronto con Elio Sgreccia
}

\section{The vision of Salvatore Privitera about the assisted reproduction debating with Elio Sgreccia}

\begin{abstract}
STRESZCZENIE:
WSPÓŁCZESNE WYZWANIA RODZĄ POTRZEBE, DOBREGO ZROZUMIENIA WARTOŚC SŁOWA W JEGO PEŁNYM ZNACZENIU, KTÓRE ZAKEADA ODPOWIEDZIALNOŚCI I TRUDNOŚCI Z JAKIM WIĄŻE SIĘ POJĘCIE SZTUCZNEGO ZAPŁODNIENIA. W POWYŻSZYM ARTYKULE ZOSTANĄ PRZEDSTAWIONE WYBRANE MYŚLI DOTYCZĄCE SPOJRzenia Salvatore Privitera Na ZagadNiEnie SZTUCZNEGO ZAPEODNIENIA. TE REFLEKSJE POCHODZA Z ANTROPOLOGII PRIVITERY JAKO FENOMENOLOGICZNO -

ANALITYCZNEGO STANOWISKA: JEST TO ETYKA

ANALITYCZNA W PERSPEKTYWIE UNIWERSALIZACJI SĄDÓW MORALNYCH, BEZ ŻADNEGO KONFLIKTU MIEDZY BIOETYKĄ „ŚWIECKĄ, LAICKĄ” A „KATOLICKĄ”. TEN PUNKT SPOJRZENIA JEST SKONFRONTOWANY ZE SPOSOBEM MYŚLENIA ELIO SGRECCIA, KTÓRY PREZENTUJE BIOETYKĘ PERSONALISTYCZNA. KONFRONTACJA (RAPORT) POMIĘDZY PRIVITERĄ I SGRECCIĄ WEDEUG WYŻEJ PRZEDSTAWIONEJ PERSPEKTYWY, BĘDZIE OKAZJĄ DO POGŁĘBIENIA SPOSTRZEŻEŃ TEOLOGICZNYCH I POKARZE JAK RADZIĆ SOBIE Z PROPOZYCJAMI Z PUNKTU WIDZENIA ŚCIŚLE ETYCZNEGO.
\end{abstract}

\section{SŁOWA KLUCZOWE:}

PERSONALIZM, SZTUCZNE ZAPEODNIENIE, SALVATORE

PRIVITERA

\section{ABSTRACT: \\ FROM A CONTEMPORARY CHALLENGE, COMES THE NEED} TO DEEP UNDERSTAND THE VALUE OF A MEANINGFUL WORD THAT, COATING OF RESPONSIBILITY, AIMS TO REPRESENT WHAT, WITH DIFFICULTY, IT IS EXPRESSED IN CONCEPT OF ARTIFICIAL INSEMINATION. IN THE PRESENT ARTICLE SOME THOUGHTS RELATED TO THIS TOPIC ARE CLEARLY Stated by Salvatore Privitera. These Reflections COME FROM A PRIVITERIANA ANTHROPOLOGY WITH A PHENOMENOLOGICAL-ANALYTICAL POSITION: AN ANALYTICAL ETHICS APPROACH FROM A PERSPECTIVE OF UNIVERSALIZATION OF MORAL JUDGMENTS, WITHOUT ANY CONFLICT, INCLUDING A PRESUMED “LAICAL” AND "CATHOlic" bioethics. This topic IS FACED With the ELIO SGRECCIA's THOUGHT, WHICH IS IN EFFECT A ONTOLOGICALLY FOUNDED PERSONALIST. THE RELATIONSHIP BETWEEN PRIVITERA ANd SGRECCIA, ACCORDING TO THE PRESENTED PERSPECTIVE, WILL PROVIDE OPPORTUNITIES FOR THEOLOGICAL INSIGHTS AND TRY TO DEAL WITH PROPOSALS ON A MORE ETHICAL LEVEL.

\section{KEYWORDS:}

PERSONALISM, ARTIFICIAL INSEMINATION, SALVATORE PRIVITERA 
$\mathrm{O}$ ggi l'uomo si trova in una società dove vince il relativismo contemporaneo ${ }^{1}$, cioè "ognuno può essere libero di poter scegliere quello che corrisponde di piů alla "sua" etica. Come se esistesse un'etica propria, di un altro o di altri ancora, di un gruppo o di un altro ancora" 2 . In questo clima la fecondazione si colloca nel desiderio personale della "realizzazione" del voler vivere l'esperienza della paternità 0 , piů frequentemente, della maternità. Detto in altri termini, esiste un diritto al figlio. Affermarlo significa che un uomo possa essere oggetto del diritto altrui perché le persone che non possono avere figli, nel modo naturale, vogliono avere il diritto di realizzare la maternità o la paternità tramite la fecondazione artificiale, ma in questo modo, viene a mancare il diritto dell' embrione, escludendo tutti quei diritti che gli appartengono.

Uno degli argomenti cruciali quindi è se la donna, come anche l' uomo, abbiano il diritto di realizzare la maternità o la paternità anche quando c'è un problema di sterilità o infertilità, attraverso le tecniche di fecondazione assistita ${ }^{3}$.

Questo pensiero del relativismo etico si afferma nel pensiero contemporaneo di alcuni italiani di questa generazione. La procreazione - evento di significato insondabile per i mille riflessi che ha in sé, per la coppia, per il concepito, per la famiglia e per la società - è diventata "un bene di consumo relativo ad altri beni di consumo; è stata ridotta ad un evento che ha dei rischi che si devono evitare e che deve, quindi, essere abbondantemente controllato. Da qui la modernizzazione dei comportamenti procreativi per avere un figlio quando, dove e come si vuole" ${ }^{\text {" }}$. Ciò è dimostrato dai sondaggi sulle persone interpellate: ${ }^{5}$ una grande percentuale di italiani (tra il 65-70\%) è a favore della fecondazione assistita, nei casi in cui le coppie non possano avere figli ${ }^{6}$.

1 Per approfondire il tema di relativismo contemporaneo vedi: S. Privitera. "Il problema del relativismo culturale di oggi”. In S. Privitera. (A cura di). Sul relativismo della cultura contemporanea. Istituto Siciliano di Bioetica Acireale 2003 s. 18; "La caratteristica del relativismo contemporaneo, consequenziale a quella delle scientificità, consiste nel fatto che esso, proprio nel nome della scientificità, risulta sotterraneamente invisibile: talvolta esso arriva ad affermare esplicitamente che Dio non esiste, o che l'etica sia senza verità, ma altrettanto frequentemente esso, sempre nel nome della scientificità, nega l'esistenza di Dio o la conoscibilità dei giudizi morali ultimi senza lasciare minimamente trapelare una simile prospettiva o presentando questa negazione come discorso altamente scientifico"; ID. "Relativismo". In S. Leone - S. Privitera. (A cura di). "Dizionario di Bioetica”. Istituto Siciliano di Bioetica Palermo $1994 \mathrm{~s}$. 824-826.

2 S. Privitera. "Quando è lecita la fecondazione artificiale”. In Bioetica e Cultura 35 (2008) s. 11: "Non è l'etica fatta per uomo, ma è l'uomo, in quanto soggetto, in quanto persona, a doversi muovere all'intero di una prospettiva etica, a dover cercare per tutte le sue azioni quel giudizio morale che corrisponde al punto di vista della imparzialità".

Ibid.

L'affermazione si trova nel articolo: P. Donati. "Trasformazioni socio-culturali della famiglia e comportamenti relativi alla procreazione". In Medicina e Morale 43 (1993) 117-163; citato in A. Serra. "Procreazione umana: la sfida tecnologica”. In Vita e Pensiero 6 (2000) s. 581-582.

5 Esempio: centro di ricerche Osserva Scienze in Society, l'Eurispes, Telefono Azzurro.

$6 \quad$ Cf. http://www.eurispes.it/index.php?option=com_content\&view=article\&id=2129: il-65-degli-italianie-a-favore-della-fecondazione-artificiale\&catid=40:comunicati-stampa\&Itemid=135 


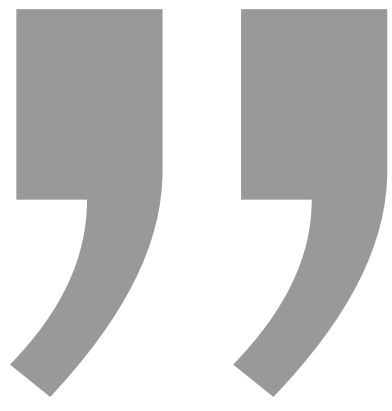

\section{E evidente che un tale argomento apra le porte al relativismo morale del pensiero.} Non esistono comportamenti sempre e comunque illeciti, ma uno stesso comportamento puo essere illecito in certe circostanze e lecito in altre. E non offrendo criteri oggettivi per valutare le conseguenze delle varie scelte, si autorizza un radicale soggettivismo del pensiero che diviene relativismo etico.

"Non è possibile imporre scelte morali e tipiche della fede religiosa a chi non le condivide" " è chiaro che coloro che si dichiarano non credenti sono piů propensi alla fecondazione assistita, anche se riconoscersi nella religione cattolica non porta necessariamente ad assumere atteggiamenti contrari rispetto alla media ${ }^{8}$.

È evidente che un tale argomento apra le porte al relativismo morale del pensiero. Non esistono comportamenti sempre e comunque illeciti, ma uno stesso comportamento può essere illecito in certe circostanze e lecito in altre. E non offrendo criteri oggettivi per valutare le conseguenze delle varie scelte, si autorizza un radicale soggettivismo del pensiero che diviene relativismo etico.

Il pensiero di Salvatore Privitera si può definire come "fenomenologicoanalitico". Usando questa definizione, egli risponde ad una esigenza che ormai da qualche decennio sollecita la riflessione morale, nel contesto cristiano, a tal punto da costituire il problema principale: come comunicare le norme morali all'interno di una società nella quale manca un consenso sulle evidenze cristiane piů fondamentali?

Per pensiero fenomenologico-analitico di Privitera si intende piů che un contenuto, una metodologia attenta non solo ai problemi della fede, ma anche alla morale e ai suoi fondamenti. Questa metodologia prevede come tappa obbligatoria, per la formulazione di qualsiasi norma morale, per qualsiasi contesto esistenziale, l'assunzione di una serie di elementi provenienti dalle scienze empiriche e di una serie

[accesso: 15-04-2016]; http://www.observa.it/view_page.aspx?ID=102\&LAN=ita [accesso: 15-04-2016].

Cf. G. Raffi, Fecondazione artificiale, http://www.parvapolis.it/page.php?id=17892 [accesso: 15-042016].

$8 \quad$ Cf. http://www.eurispes.it/index.php?option=com_content\&view=article\&id=2129: il-65-degli-italianie-a-favore-della-fecondazione-artificiale \&catid=40:comunicati-stampa\&Itemid=135 [acesso: $15-04-2016$ ]; http://www.observa.it/view_page.aspx?ID=102\&LAN=ita [accesso: 15-04-2016]. 
di strumenti concettuali ricavati dalla teoria fenomenologica dei valori e dall'analisi del linguaggio morale ordinario ${ }^{9}$.

Questa metodologia aiuta a costituire alcune distinzioni veramente capitali che emergono poi nel fare morale: permette di distinguere il piano descrittivo da quello normativo; la genesi dei giudizi morali dalla loro validità; l'etica normativa ${ }^{10}$ da quella metaetica ${ }^{11}$ e da quella parenetica ${ }^{12}$.

Secondo Privitera queste distinzioni sono fondamentali per il fatto che aiutano ad articolare meglio il rapporto tra fede e morale: se da una parte la fede non va separata dalla morale, dall'altra la fede non va confusa con la morale. Il nostro autore a questo

9 Cf. S. Privitera. Il volto morale dell'uomo. Avvio allo studio dell'etica filosofica e teologica. Istituto Siciliano di Bioetica Acireale 1999 s. 83: "Usando il metodo introspettivo, ci si accorge innanzitutto, che il fenomeno morale appare chiaramente osservabile e minuziosamente descrivibile in tutte le sue piủ caratteristiche sfumature, rilevabili in tutta la sua continuità sincronico-temporale e diacronico-geografica, come anche in tutta la sua discontinuità sincronica e diacronica. Esso appare, cioè, come fatto per certi versi sempre e dovunque identico, per altri sempre e dovunque diverso. L'identità e la diversità appaiono pure pluristratificate in una serie di cerchi concentrici e diacentrici in cui il denominatore comune risulta sempre identico e sempre diverso nella sua identità (...). La logica del discorso morale è appunto quella della discrezione: essa si identifica con quella bene precisa struttura epistemica, che consiste nella rivelazione del dato di fatto per descriverlo nel modo piů esaustivo possibile, sulla stessa scia della struttura epistemica della sociologia, della storia, della etnologia e delle scienze empiriche in genere".

S. Privitera definisce così l'etica normativa: "Nella storia dell'etica filosofica, come pure di quella teologica, si è posto spesso, in modo implicito o esplicito, il problema delle fontes moralitatis. Ponendo questo problema si cerca di sapere dove in ultima analisi vada a sedimentarsi la moralità della persona, rispondendo a domande come: quando si diventa buoni? Da dove scaturisce la moralità del soggetto morale? In che cosa consiste? Con che cosa si identifica? Rispondere a queste domande significa distinguere innanzitutto la sfera dell'atteggiamento morale o dell'atto volontario interno da quella del comportamento o dell'atto volontario esterno, per vedere se la bontà morale di una persona dipenda e si identifichi col suo atteggiamento o col suo comportamento. Normalmente per qualificare la corrispondenza dell'atteggiamento dal punto di vista della morale si usa la formula moralmente buono/moralmente cattivo, mentre per il comportamento si preferisce usare moralmente retto/moralmente errato, per distinguere anche linguisticamente il giudizio formulato sul comportamento da quello sull'atteggiamento. Con questa aggettivazione viene pure chiaramente evidenziato che l'azione diventa moralmente retta solo se e quando è strutturata ed attuata sulla base di questa conformità esteriore alla norma e che l'adesione interiore, in cui si sedimenta la moralità della persona, si identifica sempre con la conformità dell'atteggiamento. Secondo una delle tante distinzioni classiche che vengono operate in etica la bontà morale di una persona può identificarsi o dipendere dal suo comportamento moralmente retto o identificarsi e dipendere dal suo atteggiamento moralmente buono: la prima teoria dà vita a quella che viene chiamata "etica del successo", la seconda all"'etica dell'atteggiamento". Secondo l'etica dell'atteggiamento, il comportamento moralmente retto consiste nell'osservanza materiale della norma e non produce atteggiamento morale buono. Non si diventa buoni, in altri termini, perché ci si comporta in modo moralmente retto, ma perché si possiede un atteggiamento moralmente buono. Proprio perché si possiede quest’atteggiamento e perché si vuoi essere fino in fondo coerenti con esso si agisce, poi, correttamente dal punto di vista morale”, S. Privitera. "Etica normativa”. In Dizionario di Bioetica. S. Leone - S. Privitera. (A cura di). Istituto Siciliano di Bioetica Acireale 1994 s. 351-352.

11 La definizione di metaetica S. Privitera presenta cosi: "Con metaetica si intende la riflessione sui cosiddetti problemi di confine che, collocandosi al di là della riflessione di tipo normativo, 
punto si domanda se la morale si distingua dalla normativa descrittiva, metaetica e parenetica. Se descrivere non è dare delle norme, allora c'è da chiedersi: a livello normativo la fede che contributo offre $2^{13} \mathrm{Se}$, per esempio, a livello descrittivo molti sostengono l'illiceità della fecondazione artificiale, avanzando come motivo il fatto di essere cattolici, ciò significa che l'illiceità della fecondazione artificiale è giustificabile solo per una ben precisa posizione di fede?

Proprio quest'ultima domanda ci introduce nel cuore del nostro problema, oggetto della tesi. Secondo Privitera, il personalismo di Sgreccia potrebbe prestare il fianco all'idea che le norme morali abbiano bisogno, per il loro corretto processo di formulazione, della rilevanza antropologica proveniente dalla fede cristiana.

Per Privitera è piů minacciosa l'ammissione della necessità dell'antropologia cristiana per la formulazione delle norme morali, perché il rischio è quello di cadere in una specie di fideismo di stampo religioso che non rispetta in modo adeguato l'autonomia della morale ${ }^{14}$. Siamo nel cuore della questione: l'autonomia morale giustifica la metodologia fenomenologico-analitica. Che cos'è l'autonomia morale? E' un'autocomprensione della teologia morale in base alla quale non esisterebbero in campo morale verità totalmente inaccessibili alla ragione, tali per cui se è innegabile un contributo proveniente dalla fede cristiana, non è però esclusivo, ma va invece

costituiscono l'orizzonte ultimo della riflessione etica e il punto di demarcazione pure fra la riflessione puramente filosofica e quella teologica sui problemi morali. In base alla definizione data di metaetica si deve evidenziare subito come essa non sia l'equivalente di quel tipo di riflessione chiamato etica analitica, che si riferisce al modo di procedere della riflessione etica, sul piano normativo come su quello metanormativo, in contesto filosofico o anche teologico. Metaetica, invece, indica quel tipo di riflessione che, pur non mirando a fornire indicazioni normative, approfondisce la conoscenza di quelle problematiche identificabili con i postulati kantiani della moralità, con i presupposti dell'etica normativa o con le condizioni indispensabili per la retta impostazione della riflessione etica, anche di quella normativamente finalizzata", S. Privitera. "Metaetica”. In Dizionario di Bioetica. S. Leone - S. Privitera. (A cura di). Istituto Siciliano di Bioetica Acireale 1994 s. 629.

12 "Mentre l'etica normativa mira alla fondazione del giudizio morale per l'atteggiamento e per il comportamento umano, la parenesi non si prefigge scopi conoscitivi e intellettivi. L'etica normativa si rivolge all'intelligenza per far capire quale sia il vero giudizio morale, la parenesi si rivolge alla volontà, al cuore, per esortare e ammonire. L'argomentazione etica adduce i vari motivi pro o contro e perviene così alla fondazione e alla formulazione del giudizio morale, il discorso esortativo si rivolge al cuore dell'altro, soprattutto nel momento in cui questi manifesta maggiore debolezza volitiva, quando ha ripensamenti o indecisioni nei confronti della scelta morale fondamentale, quando sta per cedere di fronte ad una difficoltà o quando tenta di risollevarsi da una qualsiasi delle sue cadute. Esperendo la propria debolezza volitiva, la persona umana avverte pure continuamente il bisogno di essere incoraggiata, esortata, spronata, aiutata, rivolgendosi agli altri o facendo ricorso a tutte le proprie forze. In effetti, riflettendo attentamente su quello che quotidianamente viene esperito da ciascuna persona umana, la parenesi piů genuina e piů efficace è proprio quella che ciascuno rivolge a se stesso, mediante la propria coscienza", S. Privitera. "Parenesi". In Dizionario di Bioetica. S. Leone - S. Privitera. (A cura di). Istituto Siciliano di Bioetica Acireale 1994 s. 688-689.

13 Cf. S. Privitera. "La logica del linguaggio morale: metaetica, etica normativa e parenesi”. In Bioetica e Cultura 1 (1998) s. 83-93.

14 Cf. S. Privitera. Il volto morale dell'uomo... s. 307; vedi anche: S. Privitera. "Per una interpretazione del dibattito sull'autonomia morale”. In Rivista di Teologia Morale 48 (1980) s. 576. 
compreso e posizionato su livelli ben precisi ${ }^{15}$. Il problema dunque per Privitera è formulabile così: nella riflessione etica è determinante rapportare fede e ragione, e lo si può fare solo pensando all'esistenza di diversi livelli di analisi della stessa esperienza di fede che fornisce l'orizzonte fondativo dell'esperienza morale (piano metaetico), la causa genetica di tale esperienza (piano descrittivo), un linguaggio fertile e riccamente funzionale all'assimilazione di questa esperienza percepita nel corso dell'esistenza (piano parenetico) e richiede l'individuazione di regole con l'ausilio della ragione per la comprensibilità da parte di tutti, credenti e non credenti (piano normativo) ${ }^{16}$. L'oggetto della morale obbliga la riflessione ad analizzare la portata cognitiva di ciascun elemento, di vedere sino a dove la fede può obbligare il rispetto di una regola e se quest'ultima, in quanto perfettamente morale quindi universalizzabile, ha la fede come sua unica sorgente ${ }^{17}$. La prospettiva unica di Privitera, che è anche la sua unica preoccupazione, è la comprensibilità da parte di tutti delle norme morali.

In questo modo l'osservazione che rende pio̊ efficace la presentazione del suo pensiero è che esso ruota sempre attorno ad un'idea espressa brevemente in un enunciato che si trova in un breve suo scritto: "ciò di cui si ha bisogno in teologia oggi è che ci sia proprio la linearità logica di ogni sua fondazione epistemica" ${ }^{18}$. Questa sintetica affermazione è già un'esigenza necessaria in una delle prime sue pubblicazioni: "Non operare la distinzione dei piani significa ostacolare già la possibilità di raggiungere quella chiarezza necessaria alla condizione dell'analisi che porta alla risoluzione della problematica efacilitare il verificarsi di equivoci terminologico-concettuali in cui due affermazioni reciprocamente negantesi risultano vere" $^{\prime 19}$. La stessa impostazione ritorna praticamente nell'ultima fase della sua vita, data alla stampa dopo la sua morte, quasi a titolo di testamento: "Il rinnovamento della teologia è anche, e innanzitutto, chiarificazione logica e linearità dei suoi discorsi. Non potrà esserci, pertanto, vero rinnovamento fino a quando, nel portare avanti una riflessione, non si distinguerà nettamente il livello su cui ci si sta muovendo" ${ }^{20}$. Siamo di fronte ad un accoglimento del suo pensiero che non lascia dubbi, che diventa pressante invito a riflettere "sul significato di ogni parola, a coglierlo in ogni sua sfumatura, ad esporre la logica di ogni affermazione morale ed a comprendere

15 Cf. S. Privitera. "Autonomia/Teonomia”. In Dizionario di Bioetica. S. Leone - S. Privitera. (A cura di). Istituto Siciliano di Bioetica Acireale 1994 s. 81; S. Privitera. Il volto morale dell'uomo... s. 84-85.

16 Cf. S. Privitera. La logica del linguaggio morale: metaetica..., s. 88-89; S. Privitera. "L’insegnamento della teologia morale a Palermo". In Ho Theologos 1 (2001) s. 75

17 Ibid.; vedi anche :S. Privitera. "La questione bioetica. Riflessioni per il superamento degli itinerari relativistici”. In Bioetica e Cultura 2 (1998) s. 156-157.

18 S. Privitera. “Frammentazione in teologia morale?”. In Ho Theologos 3 (2000) s. 388.

19 S. Privitera. Dall'esperienza alla morale. Il problema "esperienza». Teologia Morale. Edi Oftes Palermo 1985 s. 10

20 S. Privitera. "Sulla logica del discorso morale tra teleologia e deontologia". In Bioetica e Cultura 1 (2007) s. 13. 
esattamente la res a cui ogni parola rinvia"21. Questo invito per gli altri è stato per lui un metodo da dover seguire sempre nell'insegnamento dell'etica filosofica e teologica che lo ha legato liberamente alla figura di chi, per primo, ha introdotto tale metodo in teologia morale, il grande filosofo Bruno Schüller.

La posizione priviteriana è una posizione fenomenlogico-analitica. Con la sua antropologia si presenta un orientamento dell'etica analitica in una prospettiva di universalizzare dei giudizi morali, senza alcuna contrasto tra una presunta bioetica "laica" e una "cattolica"22. La sua antropologia è l'etica. Privitera sostiene fondamentalmente che la vera antropologia è l'etica. "L'antropologia è scienza fondamentalmente descrittiva, mentre l'etica, quella normativa in particolare, con cui la bioetica va a identificarsi, è scienza che va a collocarsi sul piano assiologico e su questo piano attua il suo processo valutativo per formulare alla fine il giudizio morale" ${ }^{23}$. L'etica è come la chiave ermeneutica per la antropologia. Egli rappresenta nel suo studio il modo analitico per fare l'etica, attraverso un' impostazione fenomenologico analitica ${ }^{24}$. Il suo obiettivo è anche quello di chiarire i termini della questione, non tanto di risolverle, ma di spiegare le posizioni. Tutto ciò lo si rileva analizzando lo studio di Privitera dove tutti gli scritti appaiono su questo versante, pochissime volte tra le conclusioni cerca di approfondire le questioni con un' impostazione analitica. P. Cognato, il suo allievo così scrive: "Ho scelto quel modo analitico di fare etica, cosi come lui amava ripetere, tenendo a precisare sempre che tra questo "modo analitico" di fare l'etica e l'etica "analitica" vi era un abisso a tal punto che si infastidiva se qualche suo collega o qualche suo allievo lo incasellava tra gli analitici. Egli non si stancava mai di ripetere di non essere un analitico, ma un etico, filosofo e teologo e nient'altro perché l'etica non può che essere sostenuta in modo analitico, cioè in quel modo mediante il quale si è tutti invitati e,come obbligati, a riflettere sul significato di ogni parola, a coglierlo in ogni sua sfumatura, ad esplicitare la logica di ogni affermazione morale e a decifrare esattamente la res a cui ogni parola rinvia" ${ }^{25}$.

\section{La questione di personalismo}

Privitera non sviluppa alcun personalismo se per personalismo intendiamo una particolare corrente filosofica o antropologica. Se per personalismo intendiamo qualsiasi pensiero che difende la persona, allora possiamo dire che Privitera era un

21 S. Privitera. L'insegnamento della teologia... s. 66.

22 S. Privitera. "La bioetica come questione". In Rivista di Teologia Morale 129 (2001) s. 24. Per approfondire vedi: ID. "Per una bioetica non aggettivata, ma aggettivante". In Bioetica e Cultura 10 (1996) s. 133136; ID. "Cultura contemporanea e magistero morale”. In Rivista di Teologia Morale 106 (1996) s. 187190; ID. “Una politica per la vita”. In Rivista di Teologia Morale 112 (1996) s. 501-505.

S. Privitera. La questioni bioetica. Nodi problematici e spunti risolutivi. Istituto Siciliano di Bioetica Acireale 1999 s. 31.

24 Cf. P. Cognato. "Il pensiero moral-teologico". In S. Leone. (A cura di). Tra gli ulivi del Getsemani. In Supplemento al n. 27 di Bioetica e Cultura 1 (2005) s. 29.

25

Ibid. 
personalista, ma come risulta evidente non è questo il punto centrale; ciò che è cruciale invece è mostrare che l'etica non è piů etica se è personalista $o$ altro in quanto l'etica, se è etica vera, non può che stare dalla parte dei piů deboli e trattare tutti imparzialmente ${ }^{26}$.

Per Privitera il concetto di persona non è cosi rilevante. Inoltre potremmo dire che, secondo il suo pensiero, persona è ciò che un individuo non può cessare di essere senza cessare di esistere, un concetto del valore della persona che viene fatto funzionare con un linguaggio chiaro ed espressivo ${ }^{27}$. Tale merito tuttavia non toglie che il suo pensiero e la sua argomentazione sulla fecondazione artificiale si limitano solo ad esporre la situazione e gli elementi di una morale, lì dove si riesce ad accettare, in alcune situazioni, la liceità della fecondazione artificiale.

Privitera non parla mai di personalismo, ma di persona, ovvero di un uomo, che è tale in quanto è creato con quell'orizzonte morale che è innegabile. In altre parole, la persona è tale perché è uomo e l'uomo è tale perché è persona: l'uomo è persona perché è interpellabile eticamente, perché avverte l'esigenza morale. Questa interpellabilità etica si traduce nella capacità che l'uomo ha di cogliere i valori ${ }^{28}$. Ecco il cuore della teoria dei valori, secondo cui questi valori sono percepiti, ma non creati, preesistenti e non inventati. Gli autori di riferimento per Privitera sono i fenomenologi come Hartmann, Scheler, von Hildebrand, che hanno teorizzato il regno dei valori ${ }^{29}$.

Impostando così il discorso, Privitera non ha bisogno di alcun personalismo. Semplicemente nel contesto della procreazione assistita, così come in ogni altro contesto, lui non fa riferimento ad una concezione prefabbricata di persona per poi applicarla ai soggetti in questione, ma si riferisce, come impone il piano normativo, a tutti quegli elementi empirici e a una tavola di valori ${ }^{30}$; elemento empirico, nel contesto della procreazione medicalmente assistita, sono le tecniche usate e gli embrioni prodotti; i valori in gioco sono il desiderio legittimo di volere un bimbo e la vita umana embrionale. Tutto si gioca nel comprendere come il valore della vita embrionale debba sempre avere la precedenza rispetto al desiderio legittimo dei coniugi di volere un bambino. Se una tecnica può aiutare a realizzare questo desiderio senza danneggiare il diritto dell'embrione a vivere e svilupparsi, questo è lecito, altrimenti il giudizio rimane

26

27

"Il problema dell'embrione umano costituisce in bioetica uno dei casi piů emblematici. In certi casi, si sostiene che l'embrione possiede dei diritti solo se noi, come società, glieli riconosciamo; in altri, lo so considera come avente meno diritti, solo perché embrione, di chi non è piů in stato embrionale (...). Distinguere le diverse fasi di un'esistenza personale non significa poter assumere queste fasi come se fossero criteri diversi per rapportarsi al valore vita della persona. In ognuna delle diverse fasi, la persona possiede quel valore vita che, in quanto tale, deve essere sempre riconosciuto e garantito. Non c'è, in altri termini, un momento on cui la persona umana è piů persona, meritnado così piů rispetto per il valore vita che possiede, ed un altro in cui è meno persona, meritando meno attenzione da parte degli altri per lo stesso valore", S. Privitera. Una politica per la vita... s. 504.

Cf. S. Privitera. Il volto morale dell'uomo... s. 71-72.

Tutto il capito IV del suo libro: Il volto morale dell'uomo... s. 61-77.

Cf. S. Privitera. La questione bioetica. Riflessioni... s. 143. 
negativo ${ }^{31}$ perché, secondo Privitera, "l'azione della fecondazione che mira ad una vita umana non può essere mai moralmente accettabile se comporta anche quella del lasciar morire, o anche del lasciar in stato di congelamento, sia pure un solo embrione umano" 32 .

Da anni si cerca di discutere sullo statuto dell'embrione umano. Si parla di statuto perché il problema centrale è innanzitutto capire se l'embrione è persona umana e, in secondo luogo come diretta conseguenza, poter affermare tutta una serie di diritti intoccabili che si riferiscono solo alla persona. Tutte le argomentazioni, infatti, giungono a stabilire se e fino a quale punto si possa parlare di persona umana. Di qui le ipotesi secondo cui si possa trattare di persona sin dal concepimento o solo a partire dal quattordicesimo giorno dalla fecondazione.

Linterrogativo centrale è dunque il seguente: dove e quando si può parlare di persona umana è, infatti, il concetto di persona che in ultima analisi ha orientato tutte le ipotesi e ha guidato tutte le argomentazioni. è risultato così centrale questo concetto che ad esso si lega la dignità umana e i diritti intoccabili che ne seguono. Secondo Privitera bisogna guardare in un'altra direzione per non fare il gioco di quegli autori di stampo anglosassone $\mathrm{e}^{33}$ che hanno addirittura ipotizzato la possibilità che vi siano alcuni umani non persone e alcuni non umani persone, per cui il concetto di persona richiede il rispetto e il riconoscimento dell'altro. Avremmo così uomini ai quali nessuno accorda quel riconoscimento e quel rispetto dovuti agli animali verso i quali, invece, sono richiesti $^{34}$. Per queste ragioni Privitera ritiene che il concetto di persona, piů che risolvere, abbia complicato e fuorviato il discorso etico da svolgere sulla vita nascente. Qual è allora l'altra direzione verso cui guardare? Ecco il contributo specifico dell'impostazione priviteriana: l'argomentazione sulla fondamentalità della vita ${ }^{35}$.

Secondo Cognato l'embrione ha diritto allo stesso rispetto e alla stessa dignità di ogni persona. Per non poter sostenere questa idea, due sono le strategie messe in campo: dimostrare che l'embrione non è un essere umano oppure sostenere la distinzione netta tra essere umano e persona al fine di giungere ad affermare che tra i due solo il secondo è soggetto di diritti, mentre il primo è solo un elemento empirico-fattuale che dice l'appartenenza ad una tra le tante specie degli esseri viventi. Poiché dimostrare che l'embrione non appartenga agli esseri umani è molto difficile, se non impossibile, la strada piů battuta è sempre quella di negare all'embrione di essere persona fin dall'inizio del suo sviluppo e di attribuirgliela solo ad un certo momento del suo sviluppo ${ }^{36}$.

\footnotetext{
31 Cf. S. Privitera. La questione bioetica nella città oggi... s. 78-79.

32 Ibid. s. 76.

33 Per esempio Singer, Engelhardt.

34 Cf. S. Privitera. La questioni bioetica. Nodi problematici... s. 99.

35 Cf. Ibid. s. 98; P. Cognato. Prendersi cura della vita. Prospettiva etico-normativa e riflessione teologico morale sulla "fondamentalità" del valore vita. Il Platano di Ippocrate Palermo 2008 s. 52.

36

Cf. Ibid. s. 73-74.
} 

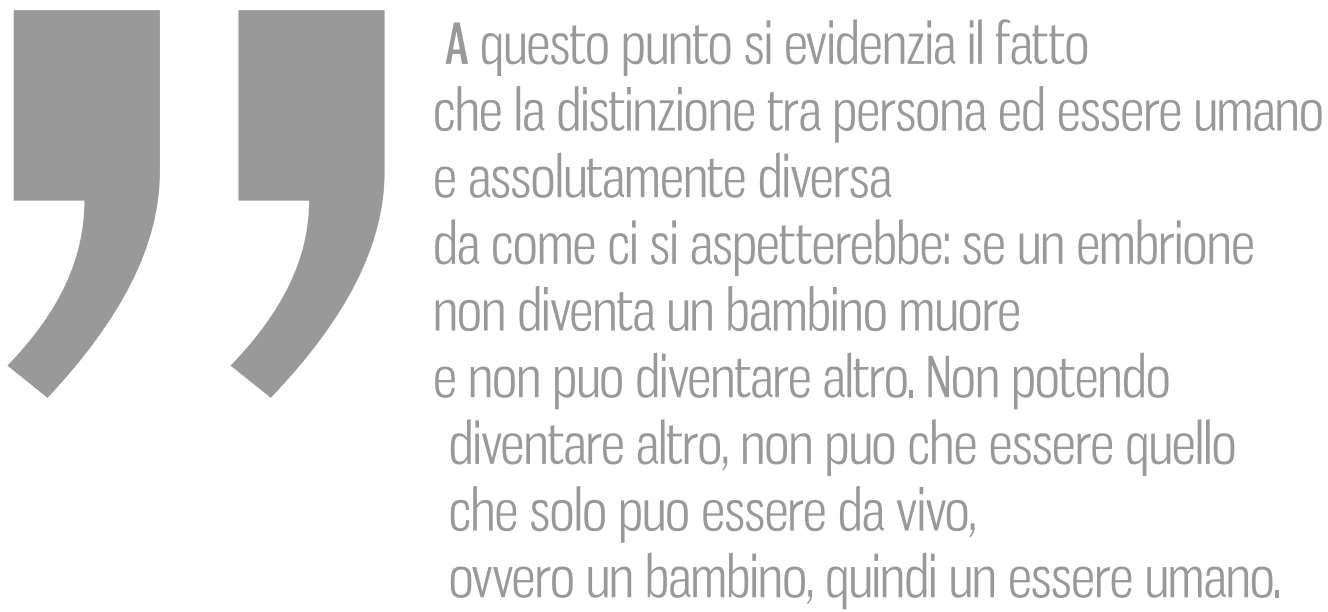

Privitera è contro la tesi che l'essere umano ha diritto al rispetto e alla vita solo quando è persona, perché l'embrione è persona in quanto è un essere umano ${ }^{37}$. Questa tesi fonda la sua plausibilità innanzitutto nel considerare assolutamente artificiosa e pretestuosa la netta distinzione tra persona ed essere umano e, in secondo luogo, nell'evidenziare piů di quanto sia già evidente la rilevanza morale della specie. Per l'etica la questione è riconoscere o no i valori, non trarre da una prefissata antropologia ciò che bisogna fare o non fare; l'etica non è antropologia.

A questo punto si evidenzia il fatto che la distinzione tra persona ed essere umano è assolutamente diversa da come ci si aspetterebbe: se un embrione non diventa un bambino muore e non può diventare altro. Non potendo diventare altro, non può che essere quello che solo può essere da vivo, ovvero un bambino, quindi un essere umano. L'argomento della vita, quale valore fondamentale, non scorge, né nella coscienza, né nell'interesse, né nella sensibilità, delle soglie significative di passaggio; al contrario, sostiene una continuità ontologica tra zigote, embrione, bambino, adulto e quindi individua solo la soglia tra l'inesistenza della novità biologica e l'esistenza della novità biologica conseguente alla fusione di due pronuclei ${ }^{38}$. Appoggiandosi su questo argomento, si stabilisce successivamente che la fondamentalità del valore vita permette di sostenere che la vita umana non può dipendere da alcuna concezione che di essa si ha, ma soltanto dal fatto che essa è il luogo dove tutti gli altri valori possono realizzarsi e che non può non essere difesa, promossa, sostenuta, aiutata, accolta, rispettata, accettata, tutelata. E non c'è niente che possa dimostrare che esiste uno stadio di preembrione o un momento in cui la vita umana non sia umana. Anche la biologia lo sostiene, ma anche se non fosse in grado di sostenerlo con certezza, una seria riflessione etica che tenga conto della dimensione valutativa oltre che descrittiva, non può che

37 Cf. S. Privitera. Una politica per la vita... s. 504.

38

Cf. P. Cognato. Prendersi cura della vita... s. 62-63. 
affermare il fatto che di fronte ad un processo continuo, articolato ed organico, come quello compreso tra il concepimento e la nascita, l'azione corretta è quella che non interrompe tale processo. Attraversare l'argomentazione della fondamentalità della vita, secondo il nostro autore, significa iniziare a pensare eticamente, senza pregiudizi concettuali, senza schieramenti filosofici, senza manifesti ideologici. La differenza per Privitera sta solo nell'etica, nell'essere parziali o imparziali, prudenti o avventati.

\section{Salvatore Privitera e la fecondazione artificiale}

Alla luce dell'argomentazione effettuata, non si rende necessaria alcuna altra riflessione personalista, se non quella minimale della interpellabilità etica dell'uomo, quando le varie metodiche comportano una strumentalizzazione o una selezione degli embrioni.

La fecondazione artificiale è una libera scelta e, pertanto, uno stato laico deve basarsi sulla morale di una parte della sua popolazione per porre divieti ad una pratica volta, fino a prova contraria, a superare i problemi di sterilità e di infertilità di una coppia $^{39}$. Secondo Privitera, grazie alle tecniche di fecondazione assistita, una coppia con un quadro di sterilità irreversibile ha il diritto fondamentale alla piena realizzazione delle vita privata familiare, perché "la procreazione assistita è da considerare sempre moralmente lecita, quando con essa si tende a procreare una nuova vita che altrimenti non sarebbe mai venuta al mondo" ${ }^{40}$. Nel contesto della fecondazione assistita Privitera, muovendosi sul piano delle diverse distinzioni ${ }^{41}$, approfondisce il problema dell'intreccio reciproco fra i valori concorrenti e le conseguenze e non il concetto di persona, formulando così un giudizio morale sulla fecondazione assistita. La tipologia omologa ed eterologa è di natura empirica e non già previamente giudicata: solo in seguito ai valori e ai disvalori delle due tipologie si può formulare un giudizio ${ }^{42}$.

Quando si parla di conseguenze, si intendono tutti i valori e i disvalori assunti imparzialmente dal moral reasoning. Pertanto per la fecondazione omologa Privitera si concentra sull'embrione, sulla selezione e sui rischi della sua struttura psico-fisica; qualora non dovessero affiorare difficoltà per la vita dell'embrione e dovesse essere superata ogni pratica selettiva odi crioconservazione embrionale, il giudizio risulterebbe lecito ${ }^{43}$.

39 Cf. Unione degli atei e degli agnostici razionalisti. Procreazione Medicalmente Assistita. www.uaar.it/laicita/procreazione_assistita.it [accesso: 10-04-2016].

40 "La liceità o la illiceità, morale di una azione umana non dipende automaticamente dal luogo biologico in cui si producono certi effetti, quanto piutossto dagli effetti, dalle conseguenze stesse che con essa vengono prodotte”. S. Privitera. La questione bioetica nella città oggi. Centro Studi Cammarata San Cataldo 1998 s. 75.

41 Parlando di distinzioni si pensa di distinguere il piano descrittivo da quello valutativo, distinguere l'argomentazione deontologica da quella teleologica, distinguere l'atteggiamento dal comportamento.

42 Cf. S. Privitera. Il volto morale dell'uomo... s. 140-141.

43 Cf. S. Privitera. "La formulazione del giudizio morale sulla fertilizzazione in vitro. Rilettura critica delle relazioni e soluzioni morale del problema”. In S. Privitera. (A cura di). La fertilizzazione in vitro. Atti del Convegno della Societas ETHICA. Palermo 2-6 settembre 1985. Edi Oftes Palermo 1986 s. 102-103. 

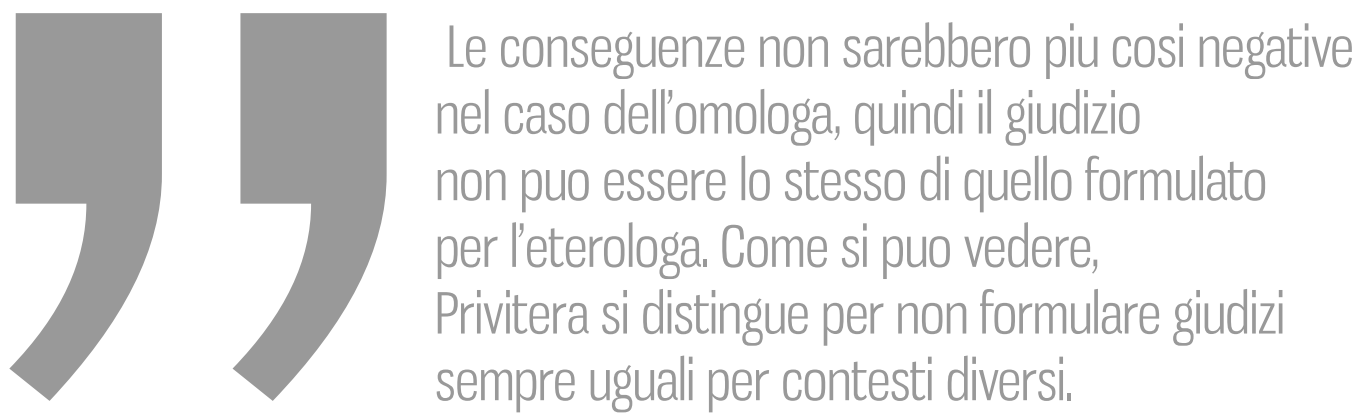

Secondo Salvatore Privitera, infatti, si possono riconoscere le tecniche di fecondazione artificiale, come moralmente accettabili, solo quando queste garantiscono la vita all'embrione, quando cioè gli sono garantiti aiuto, assistenza e serenità per una piena maturazione. L'azione della fecondazione, invece, che mira ad una vita umana, non può essere mai moralmente accettabile se comporta anche quella il lasciar morire o del lasciare in stato di congelamento sia pure un solo embrione umano ${ }^{44}$. Il bambino è sempre frutto dell'amore di due soggetti, anche quando la fecondazione avviene in modo artificiale, è cioè omologa senza sacrificare altri embrioni. Così la fecondazione assistita è concepita come una piena realizzazione e completamento di un rapporto di amore. Questo perché non si dovrebbe puntare solo sul desiderio dell'avere un figlio, come presupposto su cui fondare il ricorso alle tecniche di riproduzione assistita, ma bisognerebbe piuttosto puntare sulla genuinità e sull'autenticità del rapporto d'amore fra i due partners ${ }^{45}$. Essa diventa moralmente illecita quando il figlio viene voluto pio̊ come mezzo che come fine $\mathrm{e}^{46}$, oppure quando per avere una nuova vita, ne vengono messe al mondo altre, cioè quando altri embrioni saranno lasciati morire, senza o dopo il periodo di congelamento ${ }^{47}$. A Privitera bastano le conseguenze negative della presenza di un donatore esterno alla coppia che priverebbe il nascituro di conoscere le sue origini parentali e provocherebbe anche un caos a livello di controllo sociale, per giudicare illecita la fecondazione eterologa. ${ }^{48}$. Come è evidente qui non si tratta di andare a caccia di azioni "intrinsece malum", in quanto il problema non è se queste esistano oppure no, ma in che modo esse si possano individuare. Il criterio per Privitera rimane quello della considerazione delle conseguenze in gioco intese come valori e disvalori.

Cf. S. Privitera. La questione bioetica nella città... s. 76-77.

Cf. Ibid. s. 76-79.

"Il figlio non può mai essere frutto del loro amore quando non è, geneticamente, figlio loro: l'affermazione contraria sarebbe semplicemente una contraddizione in termini e pertanto, non avrebbe significato dal punto di vista dell'amore reciproco volere avere a tutti costi un figlio geneticamente non proprio. Essa, tuttavia, non esclude la possibilità di ricorrere all'adozione o all'affidamento, perché mediante questi istituti giuridici si tende a risanare per quanto è possibile certe situazioni negative, già verificatesi per cause "single", per via surrogata o eterologa", Ibid. s. 75-76.

Cf. Ibid. s. 76 .

Cf. Ibid.
} 
Le tecniche di fecondazione assistita, in una società secolarizzata, non assumono piů un valore il cui fondamento è Dio, ma acquistano il senso di una relazione dell'uomo con la società. Il motivo del procreare per le persone di questa società secolarizzata non è piů l'esercizio di un ministero affidato da Dio alla coppia, ma è la risposta ad un bisogno, ad un desiderio della stessa, oppure è la risposta ad una necessità sociale. Da questo punto di vista, da questa prospettiva "il figlio diventa una realtà funzionale al microcosmo familiare o al microcosmo sociale", soprattutto a vantaggio delle popolazioni tecnicamente sviluppate ${ }^{49}$.

Questo modello di pensiero si fonda "nell'impossibilità di proporre valori morali ed etici universalmente condivisi, sulla base dei quali compiere scelte e valutazioni anche nell'ambito della riproduzione e delle nuove biotecnologie; sembra dunque che si dia il via invece ad una progressiva privatizzazione della morale, legittimando la realizzazione del desiderio di un figlio a qualunque costo, in quanto mezzo di realizzazione e di gratificazione dell'adulto/genitore e della coppia” ${ }^{50}$.

Come si può notare, per Privitera, la preoccupazione costante di andare alla ricerca del vero giudizio morale sulla fecondazione artificiale passa attraverso il continuo riferirsi alle conseguenze, quelle previste e quelle prevedibili, quelle che ricadono sulla generazione presente e quelle che possono intaccare le sorti della generazione futura. E stando a questa tracciato etico-normativo il giudizio, quando la tipologia è eterologa, è decisamente negativo ${ }^{51}$. Lo squilibrio all'interno della coppia è evidente, così come è evidente la negazione del diritto del nascituro a conoscere le sue origini parentali, vista l'anonimità del donatore.

Le conseguenze non sarebbero piů così negative nel caso dell'omologa, quindi il giudizio non può essere lo stesso di quello formulato per l'eterologa. Come si può vedere, Privitera si distingue per non formulare giudizi sempre uguali per contesti diversi. è pur vero però che, guardare alle conseguenze, significa guardare a tutte le conseguenze; l'omologa potrebbe risultare illecita per Privitera solo se non si scongiura l'effetto collaterale degli embrioni sovrannumerari ${ }^{52}$. Se nella PMA sia omologa che eterologa esiste la possibilità e la realtà inevitabile della fertilizzazione di piů ovuli e del conseguente abbandono di una parte di essi, il giudizio di illiceità permane $\mathrm{e}^{53}$.

Da quanto abbiamo detto risulta evidente che la grande difficoltà da superare per Privitera è la sorte degli embrioni. In forza dell'argomento della vita, quale valore fondamentale eapplicando la regola d'oro dell'etica, il giudizio di illiceità sulla fecondazione artificiale permane. Privitera non esclude che, se si riuscisse a superare il

49 Cf. G. Muraro. "Procreazione responsabile”. In F. Compagnoni - G. Piana - S. Privitera. (A cura di). $\mathrm{Nu}$ ovo Dizionario di Teologia Morale. Edizione Paoline Milano 1990 s. 1009.

50 G. Rossi Sciumè. "La procreazione assistita: significato e problemi di un nuovo modo di diventare genitori”. In Medicina e Morale 43 (1993) s. 1144.

51 Cf. S. Privitera. Il volto morale dell'uomo... s. 140-141.

52 Cf. S. Privitera. La questione bioetica... s. 75.

53 Cf. S. Privitera. La fertilizzazione in vitro. Atti del Convegno... s. 99. 
problema degli embrioni sovrannumerari e la conseguente selezione, l'omologa non presenterebbe alcun problema, anzi attraverso di essa si realizzerebbe uno dei fini del matrimonio, ovvero la possibilità per una coppia di avere un figlio anche in una situazione problematica ${ }^{54}$. Rimane naturalmente aperta l'altra questione, quella della natura dell'atto coniugale che già in sé indicherebbe l'illiceità di tutte le tecniche di procreazione assistita, indipendentemente dalla loro tipologia omologa/eterologa ed indipendentemente dal fenomeno della sovrapproduzione degli embrioni e della loro selezione in base a criteri efficientistici.

$\mathrm{Su}$ questo argomento Privitera si inserisce proprio nel quadro di quella fondazione della norma morale che ricorre a verità in sé indiscutibile e che, nel nostro caso, è quella secondo la quale la natura ci direbbe già ciò che è giusto e ciò che non lo $\mathrm{e}^{55}$. Così come risulta illecita la contraccezione, allo stesso modo risulterebbero illecite tutte le tecniche di fecondazione assistita, per la stessa ragione che in entrambi i contesti operativi ci sarebbe una separazione tra sessualità e procreazione: se nella contraccezione vi è sessualità senza procreazione, nella fecondazione artificiale vi è procreazione senza sessualità. Quando vi è sessualità senza procreazione o vi è procreazione senza sessualità, si pone un atto contro natura, quindi illecito ${ }^{56}$. Il principio suddetto è sostenuto da considerazioni di natura antropologica e teologica. Vi è un significato antropologico del corpo in quanto esso rivela all'uomo se stesso, esprime la persona, perciò è il primo messaggio che Dio manda all'uomo. A questo significato antropologico della corporeità si aggiunge il fatto che il corpo contribuisce a rivelare l'amore che Dio ha nei confronti delle sue creature ${ }^{57}$.

Analizzando lo studio di Privitera, in conclusione si può dire che Sgreccia, e i suoi collaboratori, rappresentano il personalismo ontologicamente fondato facendo discendere dalla loro posizione personalista la spersonalizzazione dell'atto procreativo nel contesto della fecondazione assistita, essi confermano quindi maggiormente l'illiceità di tutte le tecniche per la loro forza snaturante l'atto sessuale.

Invece, secondo la prospettiva fenomenologico-analitica che presenta Privitera, molto attenta all'analisi linguistica, è che l'argomento della spersonalizzazione dell'atto procreativo o, detto in termini positivi, del significato "umano" dell'atto procreativo,

54

Cr. "Tale dottrina, più volte esposta dal magistero d che Dio ha voluto e che l'uomo non può rompere di sua iniziativa, tra i due significati dell'atto coniugale: il significato unitivo e il significato procreativo. Infatti, per la sua intima struttura, l'atto coniugale, mentre unisce con profondissimo vincolo gli sposi, li rende atti alla generazione di nuove vite, secondo leggi iscritte nell'essere stesso dell'uomo e della donna. Salvaguardando ambedue questi aspetti essenziali, unitivo e procreativo, l'atto coniugale conserva integralmente il senso di mutuo e vero amore ed il suo ordinamento all'altissima vocazione dell'uomo alla paternità. Noi pensiamo che gli uomini del nostro tempo sono particolarmente in grado di afferrare quanto questa dottrina sia consentanea alla ragione umana”, HV. 12.

57 Cf. Congregazione per l'Educazione Cattolica. Orientamenti educativi sull'amore umano. San Paolo Torino 1989 nn. 22-23; vedi anche: DV. 3 dell'introduzione EV. 43. 
è la riformulazione terminologica dell'unico vero problema, cioè sapere quale argomentazione si sta seguendo per valutare la fecondazione assistita. Negare la correttezza morale della fecondazione assistita a motivo della spersonalizzazione o della disumanizzazione dell'atto procreativo, significa seguire, in ultima analisi, le leggi della natura. La domanda corretta da porre è questa: è la natura stessa dell'uomo a fondare la norma, oppure la natura è solo un elemento tra gli altri che concorrono a fondare la norma, sebbene sia un elemento particolarmente rilevante dal punto di vista morale? L'argomentazione priviteriana che non tiene solo conto della natura dell'atto procreativo, ma che rende partecipe questo dato con altri elementi, viene non compresa quando è considerata un tentativo di rendere relative le norme morali, un modo per cercare delle eccezioni a norme che non ne possono prevedere.

Invece per Privitera solo osservando l'interezza della realtà da giudicare e quindi contemplando la possibilità che valori diversi possano entrare in concorrenza, è possibile formulare una norma pio̊ adatta e pertinente alla situazione ${ }^{58}$. Dalla prospettiva priviteriana quando si considera un certo comportamento, non lo si deve intendere come valore assoluto, come qualcosa che non può mai essere posto in concorrenza con altri valori o che, al confronto con essi, deve essere sempre considerato prioritario ${ }^{55}$. Un'azione "intrinsecamente cattiva" non può avere eccezioni perché corrisponderebbe a dire che il male in certe circostanze va compiuto. Ciò è valido anche per Privitera che ritiene ingannevole l'argomento del contro natura, in quanto se un'azione ha come conseguenze dei disvalori è a tutti gli effetti un'azione "intrinsecamente cattiva"60. Il problema, allora, per Privitera consiste proprio nel vedere se di fatto le azioni "intrinsecamente cattive" sono tali perché "contro natura". Esse esistono, ma solamente quando "in concreto" $e$ "a ragione" esse sono state riconosciute in base alla stessa realtà data e rimangono sempre tali se la realtà, precedentemente giudicata immorale, rimane immutata ${ }^{61}$.

\section{BIBLIOGRAFIA}

Cognato P. "Il pensiero moral-teologico". In S. Leone. (A cura di). Tra gli ulivi del Getsemani, in Supplemento al n. 27 di Bioetica e Cultura 1 (2005) s. 28-34.

Cognato P. Prendersi cura della vita. Prospettiva etico-normativa e riflessione teologico morale sulla "fondamentalitŕ" del valore vita. Il Platano di Ippocrate Palermo 2008.

Congregazione per l'Educazione Cattolica. Orientamenti educativi sull'amore umano. San Paolo Torino 1989.

\footnotetext{
58 Cf. S. Privitera. Dall'esperienza alla morale. Il problema... s. 238-239.

59 Cf. Ibid. s. 241.

60 Cf. Ibid. s. 233-234.

61 Cf. Ibid. s. 234.
} 
Donati P. "Trasformazioni socio-culturali della famiglia e comportamenti relativi alla procreazione”. In Medicina e Morale 43 (1993) s. 117-163.

Muraro G. "Procreazione responsabile". In F. Compagnoni - G. Piana - S. Privitera. (A cura di). Nuovo Dizionario di Teologia Morale. Edizione Paoline Milano 1990 s. 1007-1023.

Privitera S. "Autonomia/Teonomia". In Dizionario di Bioetica. S. Leone - S. Privitera. (A cura di). Istituto Siciliano di Bioetica Acireale 1994 s. 79-81.

Privitera S. "Cultura contemporanea e magistero morale". In Rivista di Teologia Morale 106 (1996) s. 187-190.

Privitera S. “Frammentazione in teologia morale?”. In Ho Theologos 3 (2000) s. 381-388.

Privitera S. "Il problema del relativismo culturale di oggi”, In S. Privitera. (A cura di). Sul relativismo della cultura contemporanea. Istituto Siciliano di Bioetica Acireale 2003 s. 13-36.

Privitera S. "L'insegnamento della teologia morale a Palermo". In Ho Theologos 1 (2001) s. 65-77.

Privitera S. "La bioetica come questione". In Rivista di Teologia Morale 129 (2001) s. 19-27.

Privitera S. "La formulazione del giudizio morale sulla fertilizzazione in vitro. Rilettura critica delle relazioni e soluzioni morale del problema”. In S. Privitera. (a cura di). La fertilizzazione in vitro. Atti del Convegno della Societas ETHICA. Palermo 2-6 settembre 1985. Edi Oftes Palermo 1986 s. 89-108.

Privitera S. "La logica del linguaggio morale: metaetica, etica normativa e parenesi”. In Bioetica e Cultura 1 (1998) s. 83-93.

Privitera S. "La questione bioetica. Riflessioni per il superamento degli itinerari relativistici”. In Bioetica e Cultura 2 (1998) s. 139-161.

Privitera S. "Etica normativa”. In Dizionario di Bioetica. S. Leone - S. Privitera. (A cura di). Istituto Siciliano di Bioetica Acireale 1994 s. 351-353.

Privitera S. "Metaetica”. In Dizionario di Bioetica. S. Leone - S. Privitera. (A cura di). Istituto Siciliano di Bioetica Acireale 1994 s. 629-632.

Privitera S. "Parenesi”. In Dizionario di Bioetica. S. Leone - S. Privitera. (A cura di). Istituto Siciliano di Bioetica Acireale 1994 s. 688-690.

Privitera S. "Relativismo". In S. Leone - S. Privitera. (A cura di). Dizionario di Bioetica". Istituto Siciliano di Bioetica Palermo 1994 s. 824-826.

Privitera S. "Per una bioetica non aggettivata, ma aggettivante". In Bioetica e Cultura 10 (1996) s. 133-136.

Privitera S. "Per una interpretazione del dibattito sull'autonomia morale". In Rivista di Teologia Morale 48 (1980) s. 565-588.

Privitera S. "Quando č lecita la fecondazione artificiale". In Bioetica e Cultura 35 (2008) s. 11-14. 
Privitera S. "Sulla logica del discorso morale tra teleologia e deontologia". In Bioetica e Cultura 1 (2007) s. 13-25.

Privitera S. "Una politica per la vita”. In Rivista di Teologia Morale 112 (1996) s. 501-505.

Privitera S. Dall'esperienza alla morale. Il problema «esperienza». Teologia Morale. Edi Oftes Palermo 1985.

Privitera S. Il volto morale dell'uomo. Avvio allo studio dell'etica filosofica e teologica. Istituto Siciliano di Bioetica Acireale 1999.

Privitera S. La questione bioetica nella cittŕ oggi. Centro Studi Cammarata. San Cataldo 1998.

Privitera S. La questioni bioetica. Nodi problematici e spunti risolutivi. Istituto Siciliano di Bioetica Acireale 1999.

Rossi Sciumč G. "La procreazione assistita: significato e problemi di un nuovo modo di diventare genitori". In Medicina e Morale 43 (1993) s. 11391169.

Serra A. "Procreazione umana: la sfida tecnologica". In Vita e Pensiero 6 (2000) S. 566-585.

http://www.eurispes.it/index.php?

option=com_content\&view=article\&id=2129: il-65-degli-italiani-e-a-

favore-della-fecondazione-artificiale\&catid=40:comunicatistampa\&Itemid $=135$

http://www.observa.it/view_page.aspx?ID=102\&LAN=ita

http://www.parvapolis.it/page.php?id=17892

http://www.eurispes.it/index.php?

option=com_content\&view=article\&id=2129: il-65-degli-italiani-e-a-

favore-della-fecondazione-artificiale\&catid=40:comunicati-

stampa\&Itemid $=135$

http://www.observa.it/view_page.aspx?ID=102\&LAN=ita

www.uaar.it/laicita/procreazione_assistita.it.

\section{OAUTORZE:}

Jacek Rosa (Siedlce) - ks. dr, prezbiter diecezji siedleckiej, bioetyk, prowadzi badania nad zagadnieniami bioetycznymi w perspektywie personalizmu chrześcijańskiego, wykładowca w Wyższej Szkole im. Bogdana Jańskiego w Warszawie, Akademia Nauk Społecznych w Warszawie. Kontakt:jacekrosa79@gmail.com 